\title{
Decreased expression of sestrin 2 predicts unfavorable outcome in colorectal cancer
}

\author{
JIN-LAI WEI ${ }^{1}$, ZHONG-XUE FU $^{1}$, MIN FANG ${ }^{2}$, JIN-BAO GUO ${ }^{1}$, QING-NING ZHAO ${ }^{3}$, \\ WEI-DONG LU ${ }^{1}$ and QIU-YUAN ZHOU ${ }^{4}$
}

\author{
Departments of ${ }^{1}$ Gastrointestinal Surgery, ${ }^{2}$ Emergency and Intensive Care Unit, The First Affiliated Hospital \\ of Chongqing Medical University, Chongqing 400016; ${ }^{3}$ Department of Pathology, Southwest Hospital, \\ Third Military Medical University, Chongqing 400038; ${ }^{4}$ Department of Pathology, Shanghai Sixth \\ People's Hospital Affiliated to Shanghai Jiao Tong University, Shanghai 200080, P.R. China
}

Received August 22, 2014; Accepted December 5, 2014

DOI: 10.3892/or.2014.3701

\begin{abstract}
Sestrin 2 is a conserved antioxidant protein that is involved in p53-dependent antioxidant defenses and protects cells against oxidative stresses. The present study was conducted to examine the expression of sestrin 2 in colorectal cancer (CRC) and investigate a possible relationship between sestrin 2 expression and prognosis in CRC. The expression of sestrin 2 in human CRC tissues and cell lines was evaluated by immunohistochemical or immunofluorescent staining and western blot analysis. The correlations between sestrin 2 expression in human CRC tissues and clinicopathological variables, including overall survival (OS) and disease-free survival (DFS), were analyzed. Both human CRC tissues and cell lines showed a decreased expression of sestrin 2 . Furthermore, a low expression of sestrin 2 was significantly correlated with advanced tumor stage, lymphatic invasion, lymph node metastasis, vascular invasion and liver metastasis. Survival analysis showed that patients with low sestrin 2 staining had a significantly worse DFS and OS. Additionally, early or advanced stage CRC patients with a low expression of sestrin 2 had a shorter survival. In univariate analysis, the patients with low sestrin 2 expression, advanced tumor stage, lymphatic invasion, lymphatic node metastasis, vascular invasion, liver metastasis and peritoneal metastasis had shorter OS and DFS. In multivariate analysis, only low sestrin 2 expression, advanced tumor stage, lymphatic node metastasis, vascular invasion and liver metastasis remained as independent prognostic factors of poor OS and DFS. The findings suggested that a decreased expression of sestrin 2 is associated
\end{abstract}

Correspondence to: Professor Zhong-Xue Fu, Department of Gastrointestinal Surgery, The First Affiliated Hospital of Chongqing Medical University, 1 You Yi Road, Chongqing 400016, P.R. China E-mail: fzx033@163.com

Key words: sestrin 2, colorectal cancer, prognosis, metastasis, survival with an unfavorable prognosis, which suggests that it is a novel and crucial predictor for CRC metastasis.

\section{Introduction}

Colorectal cancer (CRC) remains a major cause of cancer worldwide and accounts for approximately $9 \%$ of overall cancer incidence (1-2). Although recent advances in chemotherapy have prolonged the survival of patients with advanced disease, the recurrence rates remain high (3). Thus, improved understanding of CRC development may facilitate the identification of molecular targets for therapeutic intervention and improve prognosis of the disease.

Oxidative stress plays a major role in CRC development and progression (4), and results from an excess production of free radicals or insufficient antioxidant defenses. The tumor-suppressor protein p53 has received attention mainly because the gene is mutated and/or inactivated in the majority of human cancers, including CRC (5-6). p53 protein accumulation and activity are induced by genotoxic, oxidative and oncogenic stresses (7). Many p53 target genes have been thoroughly characterized and are involved in its tumor suppressive functions (8). Among these antioxidant genes activated by p53, sestrins are important for the inhibition of reactive oxygen species (ROS) and protection from oxidative stress, transformation and genomic instability (9-10). Sestrins are members of a family of highly conserved antioxidant proteins. Mammalian cells express three members of this family, including sestrin 1 , 2 and 3 (11-12). Sestrin 2, transcriptionally regulated by p53, has a cytoprotective function based on regeneration of the overoxidized peroxiredoxins (10), which are supposed to be involved in CRC (13-14). Sestrin 2 emerges as a novel player in autophagy induction and tumor suppression $(6,15)$. Upregulation of sestrin 2 expression via JNK pathway activation contributes to autophagy induction in cancer cells (16). Wang et al found that fangchinoline, a novel anti-tumor agent, induced autophagic cell death via p53/sestrin2/AMPK signalling in human hepatocellular carcinoma cells (17). Analysis of gene expression has shown that sestrin 1 and 2 are downregulated in lung cancers of different origin such as large cell carcinoma, adenocarcinoma, squamous cell carcinoma 
and small cell lung carcinoma (18-20). It is also reported that sestrin 2 interacted directly with AMPK and mediated sensitization of breast cancer cells to ionizing radiation (21). These studies suggest that the sestrin 2 may be important in tumorigenesis by regulating oxidative stress. More recently, the upregulation of sestrin 2 was found to induce apoptosis through the AMPK/p38 signaling pathway in HT-29 colon cancer cells, which are p53 mutant, treated with quercetin (22). However, the role of sestrin 2 in CRC has to be elucidated.

In the present study, we reported the expression of sestrin 2 in human CRC tissues and cell lines. The correlation between pathological factors and protein expression of sestrin 2 in human CRC tissues was examined, as well as the correlation between protein expression and disease-free survival (DFS) and overall survival (OS). To the best of our knowledge, this study provides the first evidence that sestrin 2 may be involved in CRC.

\section{Materials and methods}

Human subjects and clinical data. The parafin-embedded tissue samples of the CRC patients (130 males and 107 females) who underwent surgery between 2004 and 2008 were obtained from the Department of Gastrointestinal Surgery of the following hospitals: The First Affiliated Hospital of Chongqing Medical University and The Chongqing Three Gorges Central Hospital. The patients did not receive chemoor radiotherapy prior to sample collection. The histological type was independently determined by two pathologists in the study. The paraffin-embedded tissue specimens from 32 normal mucosa, 22 polyp, 30 adenomas ( 24 cases with mild dysplasia, 4 cases with moderate dysplasia, 2 cases with severe dysplasia) and 26 borderline tissues were used as controls. Demographics (age and gender) and tumor features (differentiation, TNM stage, lymphatic invasion, lymphatic node metastasis, invasion, liver metastasis, peritoneal metastasis and serum CEA) were obtained from clinical and pathological records (Table I). Surgical staging was determined using criteria based on International Union Against Cancer (UICC). DFS was regarded as the interval between the day that surgery was performed and the day that recurrence was identified. If recurrence was not diagnosed, the date the patient succumbed or that of last follow-up was used. OS was regarded as the interval between the dates of surgery and death. After the initial operation for the primary lesion there was a 5-year period for DFS and OS. .

Forty-two fresh CRC tissues as well as 19 normal mucosa, 20 polyp, 22 adenomas (18 cases with mild dysplasia, 3 cases with moderate dysplasia, and 1 case with severe dysplasia) and 26 borderline tissues collected between 2013 and 2014 were immediately placed in a cryovial and stored in liquid nitrogen until subsequent use for western blot analysis. Clinical features of the CRC patients are shown in Table II.

The study was approved by the Medical Ethics Review Committee of the First Affiliated Hospital of Chongqing Medical University. Informed and written consent was obtained from the patients or their relatives for the use of any data and tissues for this study. The study was performed as per the Declaration of Helsinki of the World Medical Association.
Immunohistochemistry. Tissue sections were deparaffinized in xylene, immersed in graded ethanol series, and then incubated in 3\% hydrogen peroxide for $15 \mathrm{~min}$. For the antigen retrieval, the sections were heated in a microwave oven for $10 \mathrm{~min}$ at $92-98^{\circ} \mathrm{C}$ in $10 \mathrm{mmol} / \mathrm{l}$ sodium citrate buffer ( $\mathrm{pH}$ 6.0). Non-specific binding was blocked by incubating the sections with $10 \%$ goat serum (Zhongshan Golden Bridge, Beijing, China) in 0.1 M phosphate-buffered saline (PBS) at room temperature for $30 \mathrm{~min}$ as described previously (14). The sections were then incubated with primary sestrin 2 antibody (mouse monoclonal antibody; 1:100, Santa Cruz Biotechnology, Inc., Santa Cruz, CA, USA; cat. no. sc-101249) overnight at $4^{\circ} \mathrm{C}$ followed by incubation with goat anti-mouse antibody (Zhongshan Golden Bridge, Inc., Beijing, China) for $30 \mathrm{~min}$ at $37^{\circ} \mathrm{C}$. Sections were then treated with $\mathrm{ABC}$ solution (Zhongshan Golden Bridge, Inc.) at $37^{\circ} \mathrm{C}$ for $30 \mathrm{~min}$ and washed with PBS. Immunoreactivity was detected with 3,3'-diaminobenzidine (DAB, Zhongshan Golden Bridge, Inc.) for 5 min. Counterstaining was performed using hematoxylin. For the negative controls, the primary antibodies were replaced with PBS. A LEICA DM6000B automatic-microscope (Leica, Solms, Germany) was employed for collecting of images.

The cells with buffer stain in the cytoplasm were considered to be positive. Ten random visual field images for each sample were analyzed. Staining intensity was graded on a $0-3$ scale as: 0 , absence of staining;, 1 , weakly stained; 2 , moderately stained; and 3 , strongly stained. The percentage of positive tumor cells was scored as: 0 , absence of positive cells; $1,<33 \%$ positive tumor cells; $2,33-66 \%$ positive tumor cells; and $3 ;>66 \%$ positive tumor cells. The staining score, calculated as the staining intensity score multiplied by the percentage score ranged from 0 to 9 (23). Low and high expression was regarded as a staining score of $0-4$ and 5-9, respectively. The staining score was evaluated independently by two experienced pathologists. Concordance was achieved when the two pathologists concurred on the same score for a patient. Discordant patient cases were discussed by all the pathologists from the Department of Pathology in Chongqing Medical University to reach a consensus.

Cell lines and culture conditions. The FHC human normal colorectal mucosa cell line and the human CRC HT-29, SW480, SW620 and LoVo cell lines were purchased from the Shanghai Cell Bank at the Chinese Academy of Sciences (Shanghai, China). The cell lines were cultured in Leibovitz L-15 medium (Gibco, Grand Island, NY, USA) supplemented with $10 \%$ fetal bovine serum (FBS) (Hyclone, Shanghai, China) and 2\% penicillin/streptomycin (Beyotime, Jiangsu, China). The cells were then maintained at $37^{\circ} \mathrm{C}$ in a humidified atmosphere.

Western blot analysis. Total proteins extracted from human tissues and cell lines were prepared in lysis buffer (Keygen Biotech, Nanjing, China) consisting of $50 \mathrm{mM}$ Tris (pH 7.4), $1 \%$ Triton X-100, and a protease inhibitor mixture supplemented with $1 \mathrm{mM}$ phenylmethanesulfonyl fluoride (PMSF). The insoluble material was centrifuged at $12,000 \mathrm{x} \mathrm{g}$ for $10 \mathrm{~min}$ at $4^{\circ} \mathrm{C}$, and the supernatant was obtained. The protein concentrations were quantified by bicinchoninic acid (BCA) 
Table I. Association of sestrin 2 expression with clinicopathological characteristics in 237 CRC patients.

\begin{tabular}{|c|c|c|c|c|}
\hline \multirow[b]{2}{*}{ Clinicopathological factors } & \multirow{2}{*}{$\begin{array}{l}\text { No. of patients } \\
\quad(n=237)\end{array}$} & \multicolumn{2}{|c|}{ Sestrin 2 expression } & \multirow[b]{2}{*}{ P-value } \\
\hline & & Low no. $(\%)$ & High no. (\%) & \\
\hline Age (years) & & 171 & 660.762 & \\
\hline$\geq 65$ & 104 & $74(71.2)$ & $30(28.8)$ & \\
\hline$<65$ & 133 & $97(72.9)$ & $36(27.1)$ & \\
\hline Gender & & & & 0.601 \\
\hline Male & 130 & $92(70.8)$ & $38(39.2)$ & \\
\hline Female & 107 & $79(73.8)$ & $28(26.2)$ & \\
\hline Tumor site & & & & 0.688 \\
\hline Distal & 152 & $111(73)$ & $41(27)$ & \\
\hline Proximal & 85 & 60 (70.6) & $25(29.4)$ & \\
\hline Histology & & & & 0.208 \\
\hline Well & 96 & $65(67.8)$ & $31(32.2)$ & \\
\hline Moderate/poor (mucinous) & 141 & $106(75.2)$ & $35(24.8)$ & \\
\hline TNM stage & & & & $<0.001$ \\
\hline $\mathrm{I} / \mathrm{II}$ & 78 & $43(55.1)$ & $35(44.9)$ & \\
\hline III/IV & 159 & $128(80.5)$ & $31(19.5)$ & \\
\hline Lymphatic invasion & & & & 0.004 \\
\hline Yes & 149 & 117 (78.5) & $32(21.5)$ & \\
\hline No & 88 & $54(61.4)$ & $34(38.6)$ & \\
\hline Lymph node metastasis & & & & 0.006 \\
\hline Yes & 106 & 86 (81.1) & $20(18.9)$ & \\
\hline No & 131 & $85(64.9)$ & $46(35.1)$ & \\
\hline Vascular invasion & & & & 0.012 \\
\hline Yes & 32 & $29(90.6)$ & $3(9.4)$ & \\
\hline No & 205 & $142(69.3)$ & 63 (30.7) & \\
\hline Liver metastasis & & & & 0.006 \\
\hline Yes & 35 & $32(93.5)$ & $3(6.5)$ & \\
\hline No & 202 & $139(68.9)$ & $63(31.1)$ & \\
\hline Peritoneal metastasis & & & & 0.359 \\
\hline Yes & 29 & $23(79.3)$ & $6(20.7)$ & \\
\hline No & 208 & $148(71.2)$ & $60(28.8)$ & \\
\hline Serum CEA level $(\mu \mathrm{g} / \mathrm{l})$ & & & & 0.218 \\
\hline$\geq 5$ & 175 & $130(74.3)$ & $45(25.7)$ & \\
\hline$<5$ & 62 & $41(66.1)$ & $21(33.9)$ & \\
\hline
\end{tabular}

Bold, $\mathrm{P}<0.05$.

assay (Pierce, Rockford, IL, USA). Electrophoresis was carried out using a Mini-Protean system (Bio-Rad Laboratories, Hercules, CA, USA). Total proteins $(50 \mu \mathrm{g})$ were separated on $10 \%$ SDS-PAGE gel and transferred to the polyvinylidene difluoride (PVDF) membranes (Millipore Corp., Billerica, MA, USA) by an electrophoretic transfer system (Bio-Rad Laboratories). The PVDF membranes were blocked with $5 \%$ non-fat dry milk in TBS with $0.1 \%$ Tween-20 for $1 \mathrm{~h}$ at $37^{\circ} \mathrm{C}$, and then incubated with primary antibodies, anti-sestrin 2 antibody (mouse monoclonal antibody, 1:200; Santa Cruz Biotechnology, Inc.; cat. no. sc-101249) and GAPDH antibody (mouse monoclonal antibody, 1:1,000; Abcam Biotechnology, Cambridge, MA, USA; cat. no. ab125247) overnight at $4^{\circ} \mathrm{C}$. After washing, the membranes were incubated with secondary antibodies (1:2,000 dilution, goat anti-mouse IgG-HRP; Santa Cruz Biotechnology, Inc.) for $1 \mathrm{~h}$ at $37^{\circ} \mathrm{C}$. Proteins were detected by enhanced chemiluminescence plus detection reagents (Pierce). The membranes were scanned (Bio-Rad Laboratories), and the pixel density of the images was quantified using Quantity One software (Bio-Rad Laboratories). The band intensity ratio of sestrin 2 relative to GAPDH (sestrin 2/GAPDH) was analyzed. 
Table II. Association of sestrin 2 protein expression with clinicopathological characteristics in CRC patients.

\begin{tabular}{|c|c|c|c|}
\hline $\begin{array}{l}\text { Clinicopathological } \\
\text { factors }\end{array}$ & $\begin{array}{l}\text { No. of } \\
\text { patients } \\
(\mathrm{n}=42)\end{array}$ & $\begin{array}{c}\text { Sestrin } 2 \\
\text { expression } \\
\text { protein }\end{array}$ & P-value \\
\hline Age (years) & & & 0.700 \\
\hline$\geq 65$ & 18 & $0.234 \pm 0.085$ & \\
\hline$<65$ & 24 & $0.223 \pm 0.102$ & \\
\hline Gender & & & 0.979 \\
\hline Male & 23 & $0.230 \pm 0.097$ & \\
\hline Female & 19 & $0.229 \pm 0.087$ & \\
\hline Tumor site & & & 0.815 \\
\hline Distal & 25 & $0.227 \pm 0.096$ & \\
\hline Proximal & 17 & $0.234 \pm 0.089$ & \\
\hline Histology & & & 0.340 \\
\hline Well & 14 & $0.249 \pm 0.083$ & \\
\hline $\begin{array}{l}\text { Moderate/poor } \\
\text { (mucinous) }\end{array}$ & 28 & $0.220 \pm 0.096$ & \\
\hline TNM stage & & & 0.005 \\
\hline $\mathrm{I} / \mathrm{II}$ & 13 & $0.287 \pm 0.077$ & \\
\hline III/IV & 29 & $0.204 \pm 0.087$ & \\
\hline Lymphatic invasion & & & 0.008 \\
\hline Yes & 24 & $0.198 \pm 0.084$ & \\
\hline No & 18 & $0.272 \pm 0.086$ & \\
\hline Lymph node metastasis & & & 0.012 \\
\hline Yes & 20 & $0.193 \pm 0.088$ & \\
\hline No & 22 & $0.263 \pm 0.083$ & \\
\hline Vascular invasion & & & 0.037 \\
\hline Yes & 9 & $0.174 \pm 0.072$ & \\
\hline No & 33 & $0.245 \pm 0.091$ & \\
\hline Liver metastasis & & & 0.001 \\
\hline Yes & 6 & $0.146 \pm 0.043$ & \\
\hline No & 36 & $0.244 \pm 0.090$ & \\
\hline Peritoneal metastasis & & & 0.622 \\
\hline Yes & 4 & $0.208 \pm 0.146$ & \\
\hline No & 38 & $0.232 \pm 0.087$ & \\
\hline Serum CEA level $(\mu \mathrm{g} / \mathrm{l})$ & & & 0.413 \\
\hline$\geq 5$ & 28 & $0.238 \pm 0.093$ & \\
\hline$<5$ & 14 & $0.213 \pm 0.089$ & \\
\hline
\end{tabular}

Bold, $\mathrm{P}<0.05$.

Immunofluorescence and confocal microscopy. The cells were seeded and cultured on glass coverslips the day prior to the analysis. Following incubation for $24 \mathrm{~h}$, the cells were fixed with $4 \%$ paraformaldehyde at room temperature for 15 min. After fixation, the cells were permeabilized with $0.2 \%$ Triton X-100 (Beyotime, Jiangsu, China) and blocked with $10 \%$ normal goat serum for $1 \mathrm{~h}$ at room temperature, as previously described (?). The cells were incubated with anti-sestrin 2 antibody (mouse monoclonal antibody, 1:50;
Santa Cruz Biotechnology, Inc.; cat. no. sc-101249) overnight at $4^{\circ} \mathrm{C}$. After washing with PBS, the cells were incubated with DyLight 594-conjugated goat anti-mouse IgG (1:500, Zhongshan Golden Bridge, Inc.) for $1 \mathrm{~h}$ at $37^{\circ} \mathrm{C}$. The nuclei were counterstained with DAPI (Keygen Biotech) for $10 \mathrm{~min}$, and the images were captured with an Olympus microscope (Olympus Corporation, Tokyo, Japan).

Statistical analysis. Continuous data are presented as mean \pm standard deviation (SD). Continuous variables were measured using an independent Student's t-test. The associations between sestrin 2 immunohistochemical staining and clinicopathological variables were analyzed by the Mann-Whitney U test. The log-rank test and the Kaplan-Meier analysis were used to the associations between sestrin 2 expression and the OS/DFS. Factors independently associated with OS were identified using the Cox proportional hazards model for univariate and multivariate analyses. Statistical analysis was performed using SPSS Ver. 17.0 for Windows. $\mathrm{P}<0.05$ was considered to indicate a statistically significant result.

\section{Results}

Sestrin 2 is decreased in CRC and is correlated with clinicopathological characteristics. In the normal mucosa, polyp, adenomas and borderline tissues, sestrin 2 was strongly and predominantly localized in cytoplasm, whereas faint immunoreactivity for sestrin 2 was observed in CRC patients (Fig. 1A-F). A significantly lower expression of sestrin 2 was detected in the CRC group as compared to the normal mucosa, polyp, adenomas and borderline groups respectively ( $\mathrm{P}<0.05$; Fig. $1 \mathrm{G})$. No significant difference was identified among the normal mucosa, polyp, adenomas and borderline groups ( $\mathrm{P}>0.05$; Fig. $1 \mathrm{G})$. In the $\mathrm{CRC}$ group, $72.2 \%$ of cases exhibited a low expression of sestrin 2 and $27.8 \%$ a high expression (Fig. 1H). By contrast, sestrin 2 expression was high in the normal mucosa $(87.5 \%)$, polyp (71.8\%), adenomas (80\%) and borderline (80.8\%) samples (Fig. 1H). The Mann-Whitney U test was used to evaluate whether a low expression of sestrin 2 in CRC samples was associated with specific clinicopathological variables (Table I). A low expression of sestrin 2 was significantly associated with TNM stage $(\mathrm{P}<0.001)$, lymphatic invasion $(\mathrm{P}=0.004)$, lymph node metastasis $(\mathrm{P}=0.006)$, vascular invasion $(\mathrm{P}=0.012)$ and liver metastasis $(\mathrm{P}=0.006)$. However, no significant associations were found between sestrin 2 expression and age, gender, tumor site, histology, peritoneal metastasis and serum carcinoembryonic antigen (CEA) level, respectively (all $\mathrm{P}>0.05$ ).

Correlation between the protein level of sestrin 2 and clinicopathological characteristics. Western blot analysis was performed to evaluate the sestrin 2 protein level from frozen tissues of 42 CRC patients and controls including 19 normal mucosa, 20 polyp, 22 adenomas and 26 borderline (Fig. 2). The sestrin 2 expression was strong in the normal mucosa, polyp, adenomas and borderline samples, while it was faint in CRC samples. The protein expression of sestrin 2 in CRC tissues was significantly lower than that in normal mucosa, polyp, adenomas and borderline groups $(\mathrm{P}<0.05)$. No statistical significance was found among normal mucosa, polyp, 

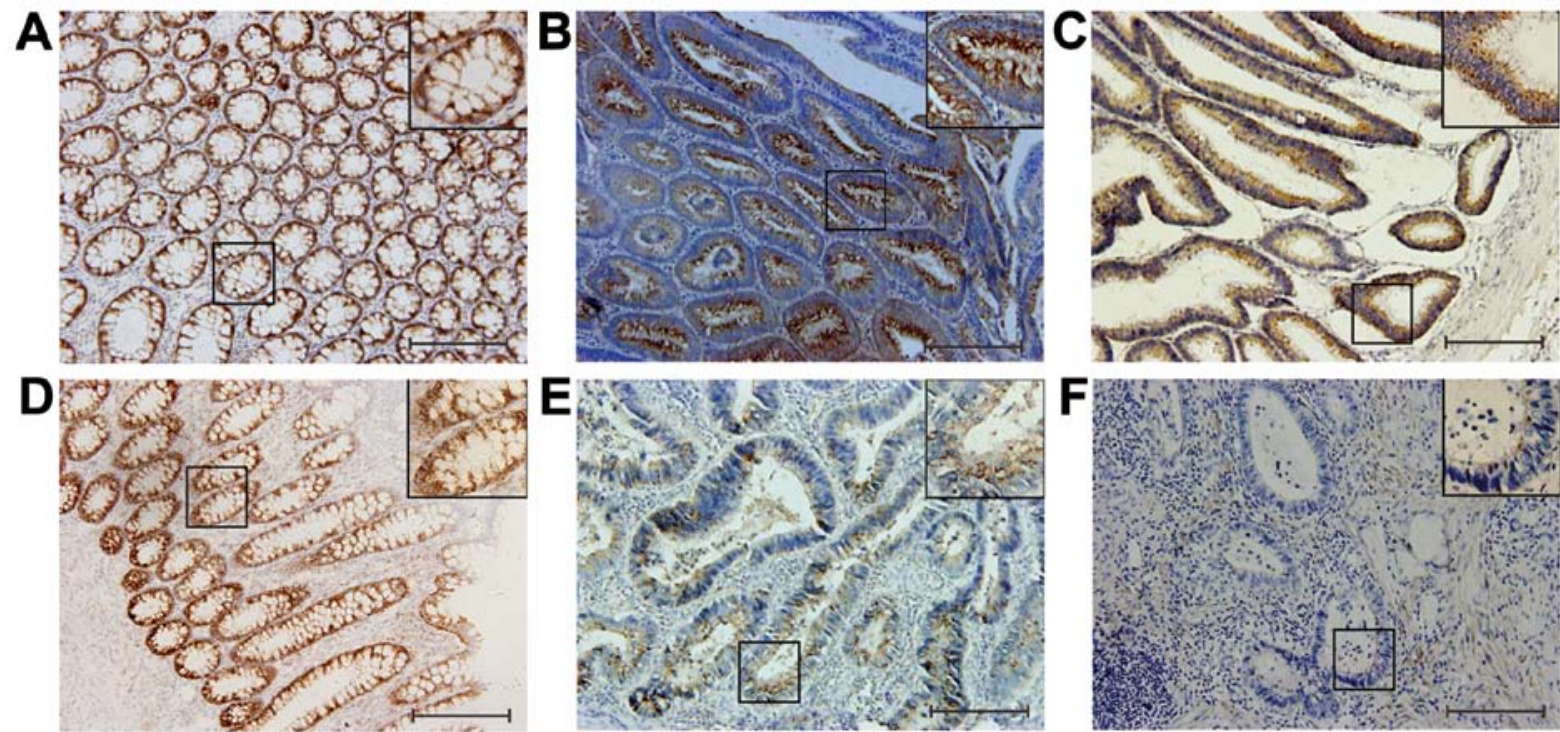

G
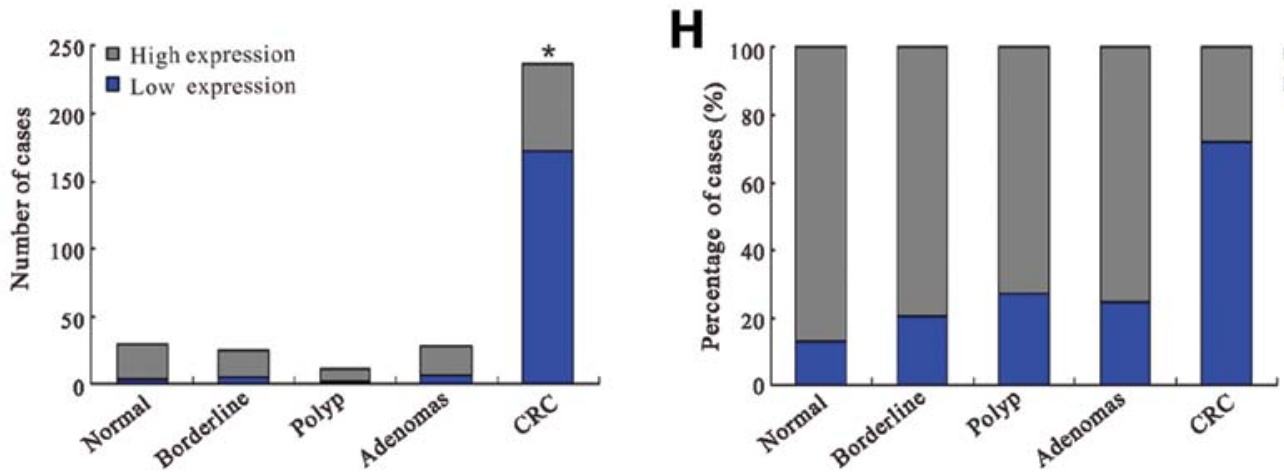

High expression

- Low expression

Figure 1. Expression of sestrin 2 in the CRC samples and controls using immunohistochemical staining. Sestrin 2 staining was mainly observed in the cytoplasm of (A) the normal mucosa, (B) polyp, (C) adenomas and (D) borderline and (E and F) CRC tissues. (G) The number of case and (H) the percentage of case showed that the proportion of low expression of sestrin 2 was higher in the CRC group than that in the normal mucosa, adenomas, polyp and borderline groups. ${ }^{*} \mathrm{P}<0.05$, statistically significant differences between different control groups and the CRC group. Scale bar, $100 \mu \mathrm{m}$.
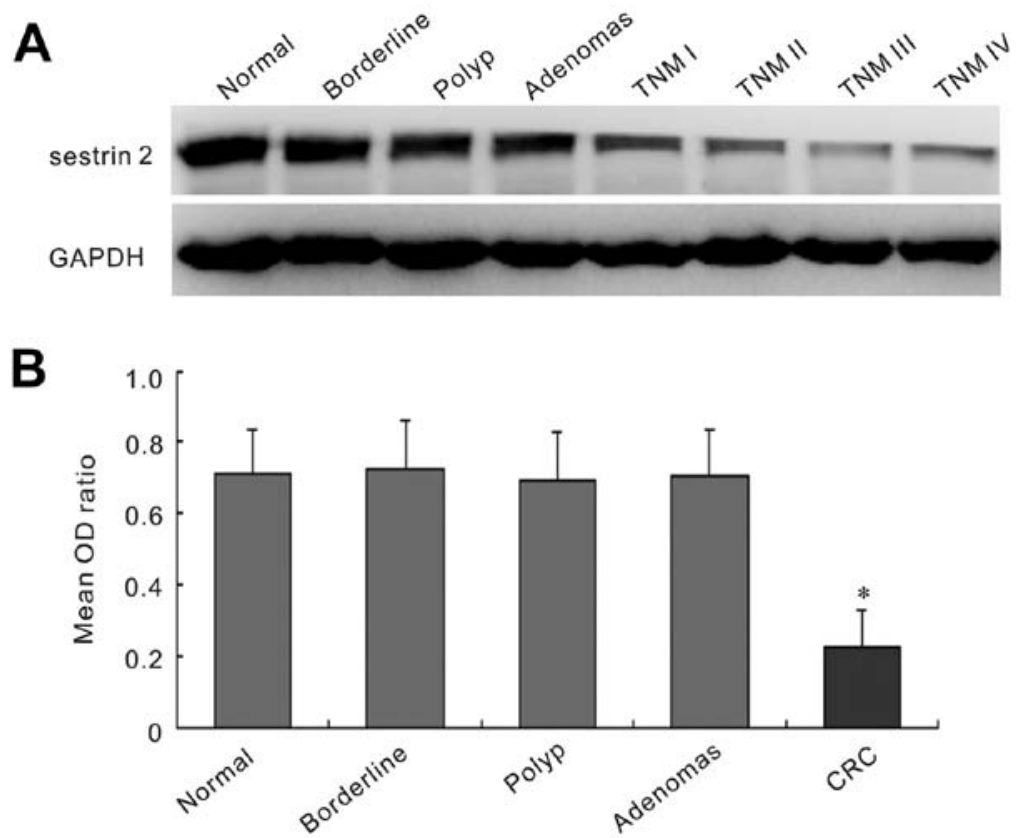

Figure 2. Western blot analysis of sestrin 2 expression in the CRC samples and controls. Representative western blotting showing sestrin 2 expression was strong in the normal mucosa, polyp, adenomas and borderline samples, while it was faint in (A) CRC samples. Histogram of the normalized sestrin 2 mean OD ratio for the (B) normal mucosa, polyp, adenomas, borderline and CRC samples. The mean OD ratio shows the density of sestrin 2 immunoreactivity relative to GAPDH immunoreactivity. ${ }^{*} \mathrm{P}<0.05$, statistically significant differences between different control groups and the CRC group. 


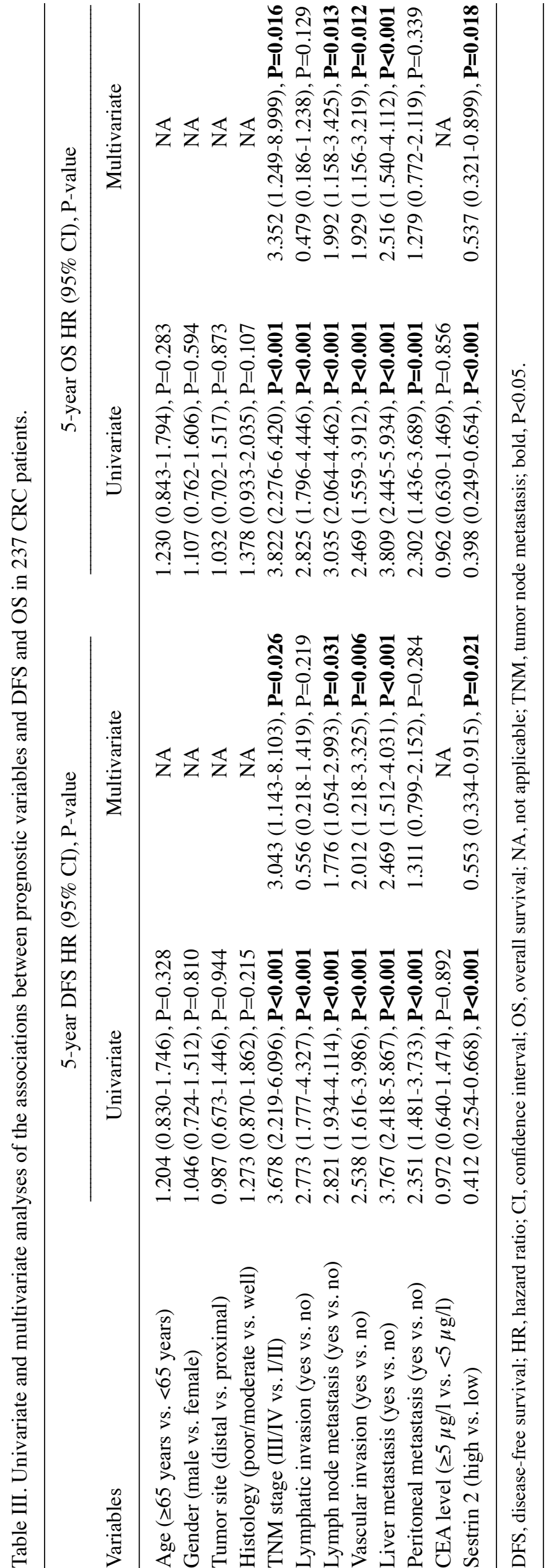

adenomas and borderline groups ( $\mathrm{P}>0.05)$. Furthermore, we analyzed the correlation between the protein level of sestrin 2 and clinicopathological characteristics. The results positively correlated with the immunohistochemical findings. A significantly lower sestrin 2 protein level was associated with TNM stage $(\mathrm{P}=0.005)$, lymphatic invasion $(\mathrm{P}=0.008)$, lymph node metastasis $(\mathrm{P}=0.012)$, vascular invasion $(\mathrm{P}=0.037)$ and liver metastasis $(\mathrm{P}=0.001)$ (Table II).

Sestrin 2 expression in colon normal mucosa and CRC cell lines. The control cell line FHC and the HT-29, SW480, SW620 and LoVo human CRC cell lines were selected to analyze the expression of sestrin 2 at the protein level by immunofluorescence and western blot analysis. Immunofluorescence showed the expression of sestrin 2 was localized mainly in cytoplasm (Fig. 3A). Sestrin 2 expression was strong in the FHC cells and moderate in HT-29 and SW480 cells, while it was faint in the SW620 and LoVo cells (Fig. 3A). Western blot analysis also revealed a markedly strong expression of sestrin 2 in FHC group and a moderate expression in the HT-29 and SW480 groups, but a markedly weak expression in the SW620 and LoVo groups (Fig. 3B). Compared with the FHC group, the expression of sestrin 2 was significantly lower in the HT-29, SW480, SW620 and LoVo groups (P<0.05; Fig. 3C). Furthermore, the expression of sestrin 2 in the SW620 and LoVo cells was significantly lower than that in the HT-29 and SW480 cells $(\mathrm{P}<0.05$; Fig. 3C).

Low expression of sestrin 2 in CRC predicts an unfavorable outcome. The correlation of sestrin 2 low expression and clinical outcome was analyzed. Following 5-year follow-up, the mean OS and DFS periods were 39.04 \pm 22.46 and $38.70 \pm 22.56$ months, respectively. To assess sestrin 2 as a predictor of survival, the Kaplan-Meier analysis method was used to investigate the correlation between sestrin 2 expression and survival. The log-rank test showed that patients with low sestrin 2 staining had a significantly worse OS and DFS than patients with high sestrin 2 staining $(\mathrm{P}<0.001$ and $\mathrm{P}<0.001$, respectively; Fig. 4A and B). Additionally, patients with early or advanced stage CRC with low expression of sestrin 2 had a shorter survival than patients with high expression $(\mathrm{P}=0.029$ and $\mathrm{P}=0.023$, respectively; Fig. $4 \mathrm{C}$ and Fig. 4D). At the 5-year follow-up, $50.99 \%$ of the patients with high sestrin 2 level survived. However, only $37.67 \%$ of patients with low sestrin 2 staining survived.

The univariate analysis showed that the patients with low sestrin 2 expression [DFS, hazard ratio $(\mathrm{HR})=0.412, \mathrm{P}<0.001$; OS, HR $=0.398, P<0.001]$, advanced tumor stage (DFS, $\mathrm{HR}=3.678, \mathrm{P}<0.001 ;$ OS, HR $=3.822, \mathrm{P}<0.001)$, lymphatic invasion (DFS, HR $=2.773, \mathrm{P}<0.001$; OS, $\mathrm{HR}=2.825$, $\mathrm{P}<0.001$ ), lymph node metastasis (DFS, $\mathrm{HR}=2.821, \mathrm{P}<0.001$; $\mathrm{OS}, \mathrm{HR}=3.035, \mathrm{P}<0.001$ ), vascular invasion (DFS, $\mathrm{HR}=2.538$, $\mathrm{P}<0.001$; OS, HR $=2.469, \mathrm{P}<0.001$ ), liver metastasis (DFS, $\mathrm{HR}=3.767, \mathrm{P}<0.001$; OS, HR $=3.809, \mathrm{P}<0.001$ ) and peritoneal metastasis (DFS, HR $=2.351, \mathrm{P}<0.001$; OS, $\mathrm{HR}=2.302$, $\mathrm{P}=0.001$ ) had shorter OS and DFS (Table III). Furthermore, the multivariate analysis showed that only low sestrin 2 expression (DFS, HR $=0.553, \mathrm{P}=0.021$; OS, $\mathrm{HR}=0.537$, $\mathrm{P}=0.018$ ), advanced TNM stage (DFS, HR $=3.043, \mathrm{P}=0.026$; $\mathrm{OS}, \mathrm{HR}=3.352, \mathrm{P}=0.016$ ), lymphatic node metastasis (DFS, 
A

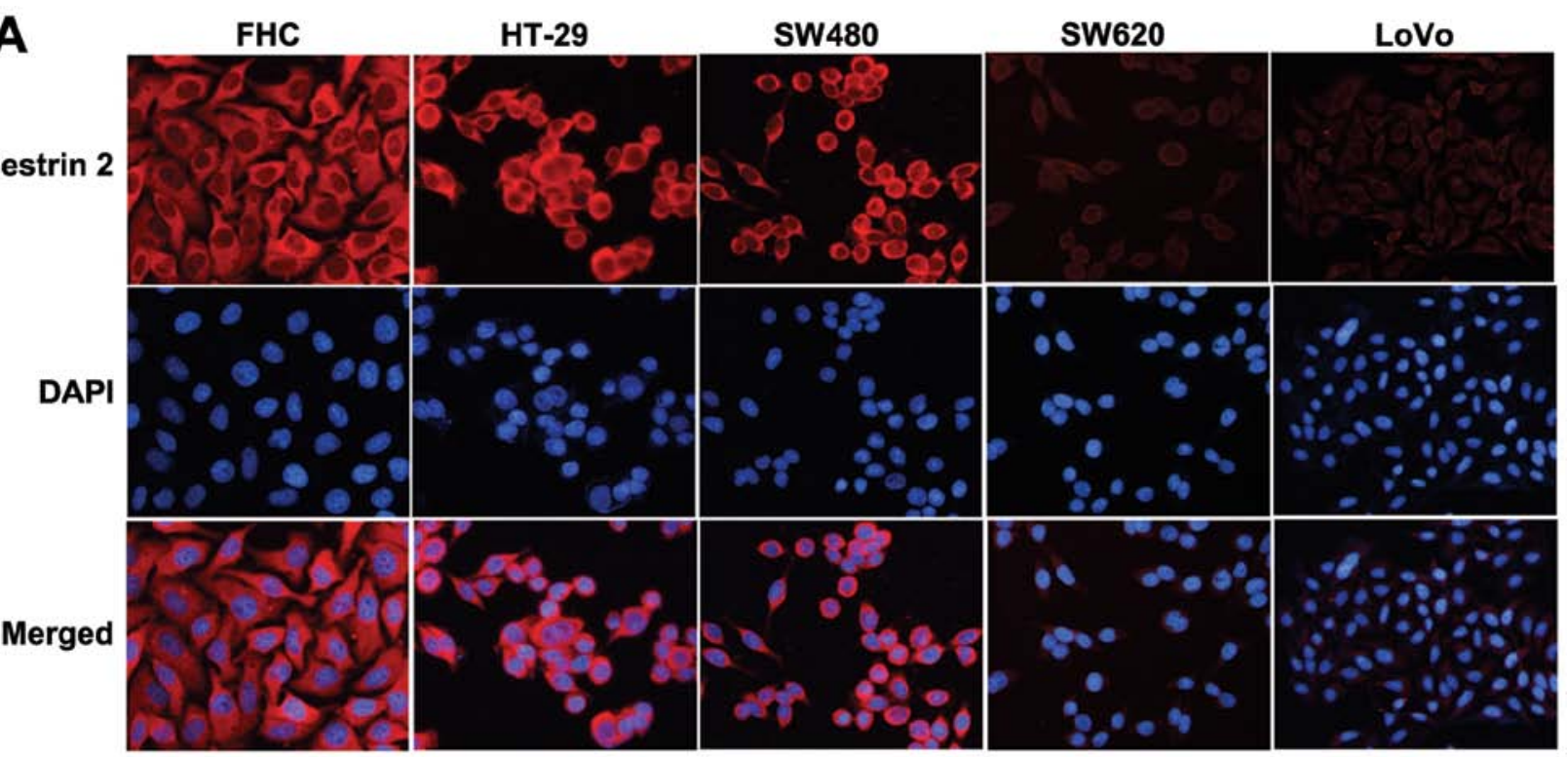

B
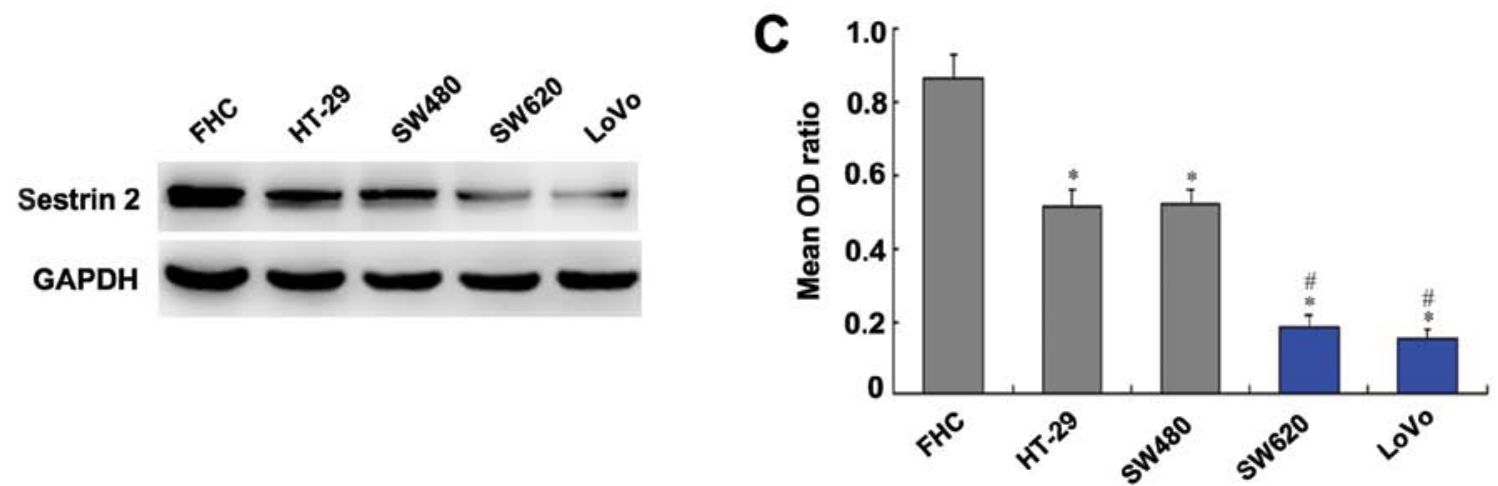

Figure 3. Immunofluorescence and western blot analysis of sestrin 2 expression in colon normal mucosa and CRC cell lines. (A) Immunofluorescence showed sestrin 2 expression was strong in the FHC cells and moderate in HT-29 and SW480 cells, while it was faint in the SW620 and LoVo cells. Sestrin 2 was marked in red, and the nuclei were marked in blue with DAPI. (B) Representative western blotting showing sestrin 2 expression was strong in the FHC group and moderate in HT-29 and SW480 groups, while it was faint in the SW620 and LoVo groups. (C) Histogram of the normalized sestrin 2 mean OD ratio for the FHC, HT-29, SW480, SW620 and LoVo groups. The mean OD ratio shows the density of sestrin 2 immunoreactivity relative to GAPDH immunoreactivity. ${ }^{*} \mathrm{P}<0.05$, statistically significant differences between the FHC group and the different $\mathrm{CRC}$ groups. ${ }^{*} \mathrm{P}<0.05$, statistically significant differences when compared with the HT-29 and SW480 groups, respectively.

$\mathrm{HR}=1.776, \mathrm{P}=0.031$; OS, $\mathrm{HR}=1.992, \mathrm{P}=0.013$ ), vascular invasion (DFS, HR =2.012, $\mathrm{P}=0.006$; OS, $\mathrm{HR}=1.929, \mathrm{P}=0.012$ ) and liver metastasis $(\mathrm{DFS}, \mathrm{HR}=2.469, \mathrm{P}<0.001$; OS, $\mathrm{HR}=2.516$, $\mathrm{P}<0.001)$ remained independent prognostic factors of poor OS and DFS (Table III). However, no significant correlation was detected between survival and other clinicopathological variables including age, gender, tumor site, histology, lymphatic invasion, peritoneal metastasis and serum CEA level (all $\mathrm{P}>0.05$; Table III).

\section{Discussion}

ROS, which are thought to be a major source of endogenous DNA damage, directly contribute to tumor progression and metastasis (24-26). A great deal of evidence support the view that oxidative stress and the accompanying ROS are genotoxic and may contribute to the development of CRC (27). Furthermore, the genetic reduction of mitochondrial oxidative stress reduces tumor grade and inhibits metastasis (28). Therefore, tumors occur when there is an imbalance between overproduction of ROS and a decrease of antioxidant molecules in the body.

In the present study, we showed that the antioxidant protein sestrin 2 was decreased in human CRC tissues. Similarly, sestrin 2 was downregulated in human CRC cell lines. The expression of sestrin 2 in SW620 and LoVo cells, which were derived from the metastatic site of CRC, was significantly lower than that in the HT-29 and SW480 cells, which were derived from the primary lesion of CRC. In subsequent analysis of the association between the sestrin 2 expression and clinicopathological variables, we found that the low expression of sestrin 2 was correlated with lymph node and liver metastasis. The findings reveal that there may be a connection between the decreased expression of sestrin 2 and tumor metastasis. Results of studies have shown that oxidative stress directly contributes to tumor progression and metastasis $(28,29)$. In clinical findings, most current chemotherapy agents and radiation therapy increase oxidative stress, leading to tumor recurrence and metastasis (28). Since sestrin 2 protect cells from oxidative stress, the 
A Patients with CRC

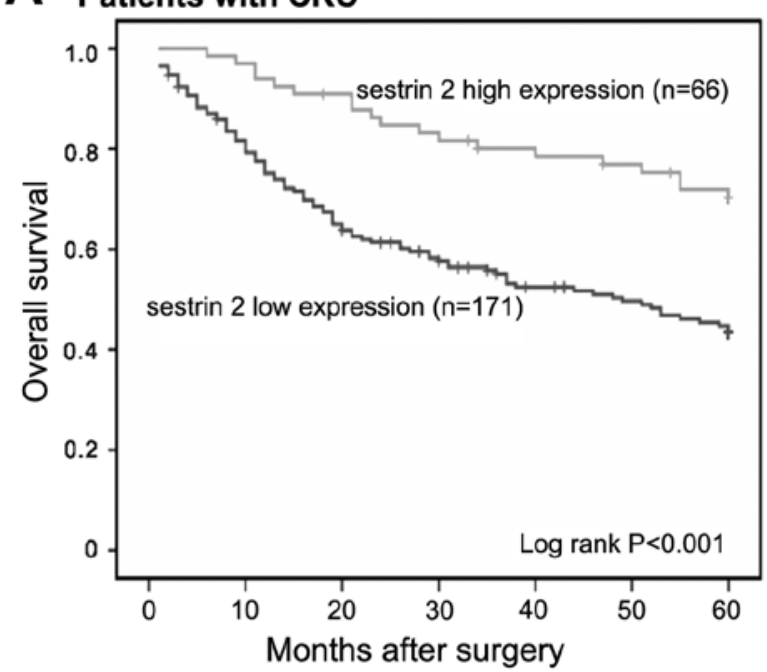

C Patients with stage I/II CRC

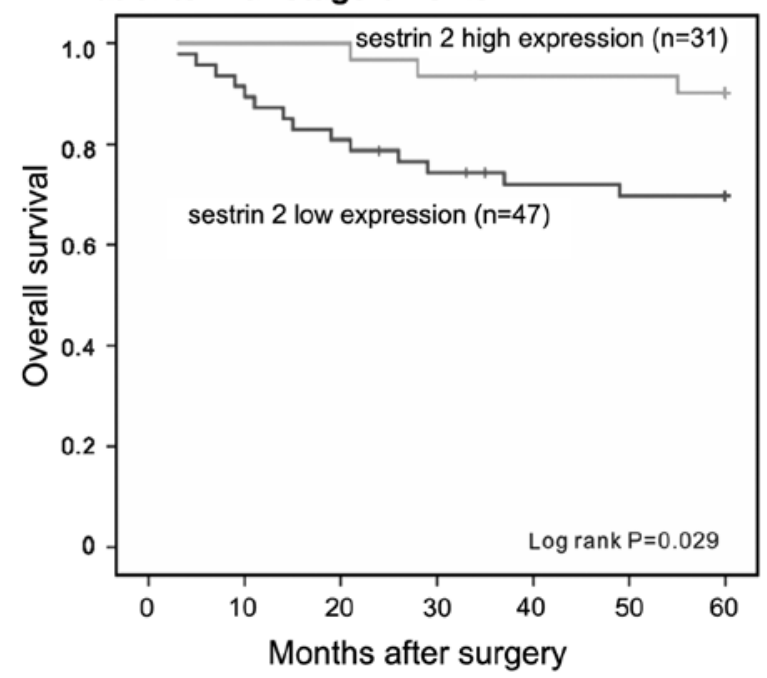

B Patients with CRC

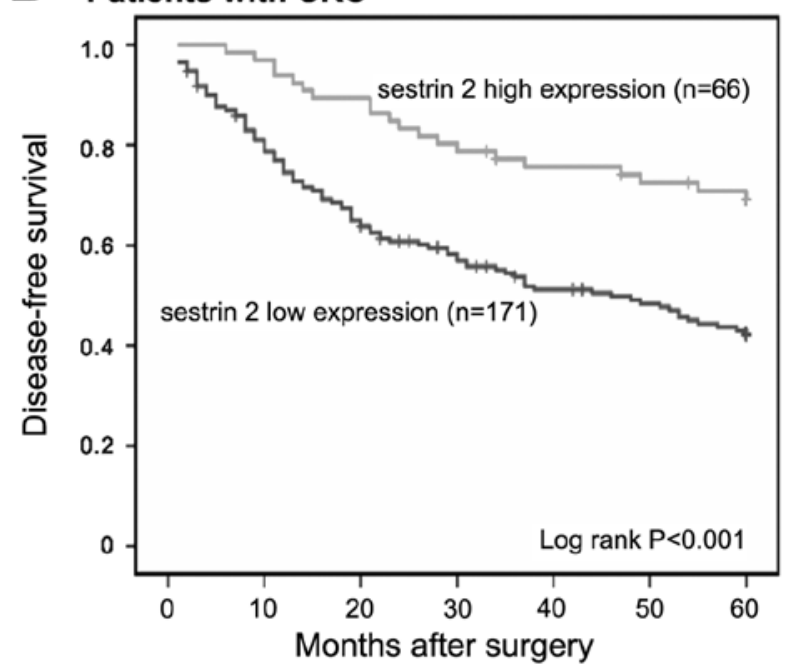

D Patients with stage III/IV CRC

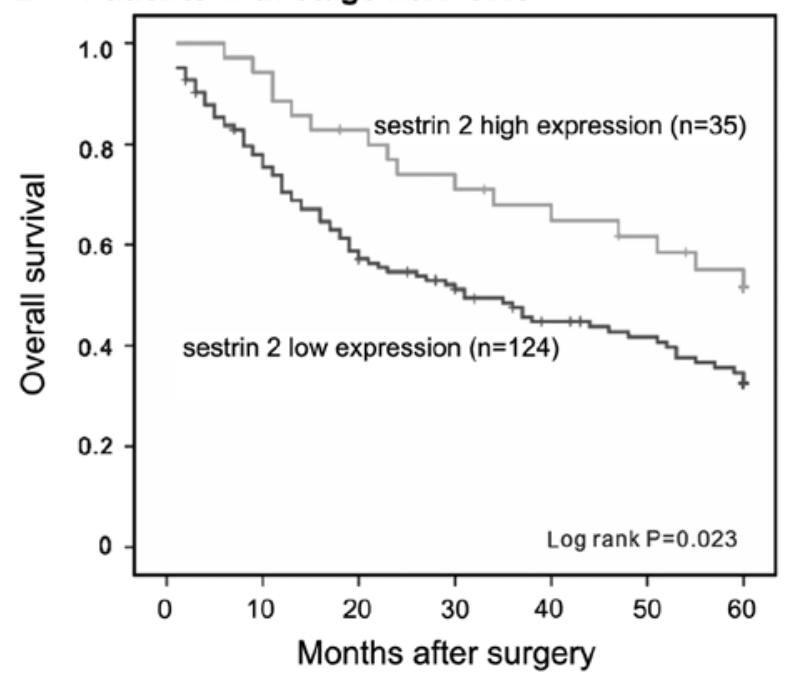

Figure 4. Kaplan-Meier analysis for the correlation between sestrin 2 expression and survival. The (A) OS and (B) DFS of CRC patients with a high and low expression of sestrin 2 are shown, respectively. Log-rank test showed that patients with high sestrin 2 staining had a significantly better OS and DFS versus patients with low sestrin 2 staining. The (C) stage I/II survival and (D) III/IV survival of CRC patients with high and low expression of sestrin 2, respectively are shown. Log-rank test showed that patients with a low expression level of sestrin 2 in stage I/II and stage III/IV had a shorter outcome.

downregulation of sestrin 2 may increase oxidative stress, thus aggravating tumor metastasis. However, the molecular pathway that connects downregulation of sestrin 2 to the acquisition of metastatic capacity during tumor progression remains to be investigated. Besides the lymph node and liver metastasis, we found that low expression of sestrin 2 was significantly correlated with advanced tumor stage, lymphatic invasion and vascular invasion.

Previous studies reported that the abnormalities of sestrin 2-related protein, p53, was associated with CRC patient survival (30-33). However, the association between sestrin 2 and cancer mortality has not been investigated in clinical samples. Our results clearly demonstrate that a decreased expression of sestrin 2 was an independent and significant prognostic factor for 5-year DFS and OS. Additionally, early or advanced stage CRC patients with a low expression of sestrin 2 had a shorter survival than patients with a high expression. To the least of our knowledg, this is the first study to show an association between sestrin 2 expression and CRC patient survival. These findings suggest that sestrin 2 and its associated proteins may be crucial in CRC patient prognosis.

In conclusion, our study of patients with CRC revealed the downregulation of sestrin 2 protein in human CRC tissues compared with the normal mucosa, polyp, adenomas and borderline tissues. The expression of sestrin 2 was decreased in HT-29, SW480, SW620 and LoVo human CRC cell lines when compared with the FHC control cell line. Additionally, decreased sestrin 2 was associated with an unfavorable prognosis and was an independent prognostic factor for CRC, suggesting that sestrin 2 is a crucial predictor for sestrin 2 metastasis. The results thus shed light on the potential of sestrin 2 as a tumor-suppressor gene with a novel antioxidant function in $\mathrm{CRC}$, and that downregulation of sestrin 2 may aggravate tumor metastasis. However, future studies should be conducted to examine the effects of changing sestrin 2 activity and identify the possible mechanisms based on this novel protein involved in CRC. 


\section{Acknowledgements}

This study was supported by the National Natural Science Foundation of China (no. 81172295 and no. 81401070). The authors sincerely thank the patients and their families for their participation in this study.

\section{References}

1. Haggar FA and Boushey RP: Colorectal cancer epidemiology: incidence, mortality, survival, and risk factors. Clin Colon Rectal Surg 22: 191-197, 2009.

2. Siegel R, Naishadham D and Jemal A: Cancer statistics for Hispanics/Latinos, 2012. CA Cancer J Clin 62: 283-298, 2012.

3. Wolpin BM and Mayer RJ: Systemic treatment of colorectal cancer. Gastroenterology 134: 1296-1310, 2008.

4. Kovacic P and Jacintho JD: Mechanisms of carcinogenesis: focus on oxidative stress and electron transfer. Curr Med Chem 8: 773-796, 2001.

5. Rodrigues NR, Rowan A, Smith ME, et al: p53 mutations in colorectal cancer. Proc Natl Acad Sci USA 87: 7555-7559, 1990.

6. Sablina AA, Budanov AV, Ilyinskaya GV, Agapova LS Kravchenko JE and Chumakov PM: The antioxidant function of the p53 tumor suppressor. Nat Med 11: 1306-1313, 2005.

7. Vousden KH and Ryan KM: p53 and metabolism. Nat Rev Cancer 9: 691-700, 2009.

8. Green DR and Kroemer G: Cytoplasmic functions of the tumour suppressor p53. Nature 458: 1127-1130, 2009.

9. Budanov AV and Karin M: p53 target genes sestrin1 and sestrin2 connect genotoxic stress and mTOR signaling. Cell 134: 451-460, 2008.

10. Budanov AV, Sablina AA, Feinstein E, Koonin EV and Chumakov PM: Regeneration of peroxiredoxins by p53-regulated sestrins, homologs of bacterial AhpD. Science 304: 596-600, 2004.

11. Velasco-Miguel S, Buckbinder L, Jean P, et al: PA26, a novel target of the p53 tumor suppressor and member of the GADD family of DNA damage and growth arrest inducible genes. Oncogene 18: 127-137, 1999.

12. Budanov AV, Shoshani T, Faerman A, et al: Identification of a novel stress-responsive gene Hi95 involved in regulation of cell viability. Oncogene 21: 6017-6031, 2002.

13. Lu W, Fu Z, Wang H, Feng J, Wei J and Guo J: Peroxiredoxin 2 knockdown by RNA interference inhibits the growth of colorectal cancer cells by downregulating Wnt/ $\beta$-catenin signaling. Cancer Lett 343: 190-199, 2014.

14. Lu W, Fu Z, Wang H, Feng J, Wei J and Guo J: Peroxiredoxin 2 is upregulated in colorectal cancer and contributes to colorectal cancer cells' survival by protecting cells from oxidative stress. Mol Cell Biochem 387: 261-270, 2014

15. Maiuri MC, Malik SA, Morselli E, et al: Stimulation of autophagy by the p53 target gene Sestrin2. Cell Cycle 8: 1571-1576, 2009.

16. Zhang XY, Wu XQ, Deng R, Sun T, Feng GK and Zhu XF: Upregulation of sestrin 2 expression via JNK pathway activation contributes to autophagy induction in cancer cells. Cell Signal 25: $150-158,2013$.
17. Wang N, Pan W, Zhu M, Zhang M, Hao X, Liang G and Feng Y: Fangchinoline induces autophagic cell death via p53/sentrin2/AMPK signaling in human hepatocellular carcinoma cells. Br J Pharmacol 164: 731-742, 2011.

18. Garber ME, Troyanskaya OG, Schluens K, et al: Diversity of gene expression in adenocarcinoma of the lung. Proc Natl Acad Sci USA 98: 13784-13789, 2001.

19. Wachi S, Yoneda K and Wu R: Interactome-transcriptome analysis reveals the high centrality of genes differentially expressed in lung cancer tissues. Bioinformatics 21: 4205-4208, 2005.

20. Su LJ, Chang CW, Wu YC, et al: Selection of DDX5 as a novel internal control for Q-RT-PCR from microarray data using a block bootstrap re-sampling scheme. BMC Genomics 8: 140, 2007.

21. Sanli T, Linher-Melville K, Tsakiridis T and Singh G: Sestrin2 modulates AMPK subunit expression and its response to ionizing radiation in breast cancer cells. PLoS One 7: e32035, 2012.

22. Kim GT, Lee SH, Kim JI and Kim YM: Quercetin regulates the sestrin 2-AMPK-p38 MAPK signaling pathway and induces apoptosis by increasing the generation of intracellular ROS in a p53-independent manner. Int J Mol Med 33: 863-869, 2014.

23. Au CW, Siu MK, Liao X, et al: Tyrosine kinase B receptor and BDNF expression in ovarian cancers - Effect on cell migration, angiogenesis and clinical outcome. Cancer Lett 281: 151-161, 2009.

24. Lee DJ and Kang SW: Reactive oxygen species and tumor metastasis. Mol Cells 35: 93-98, 2013.

25. Nishikawa M: Reactive oxygen species in tumor metastasis. Cancer Lett 266: 53-59, 2008.

26. Wu WS: The signaling mechanism of ROS in tumor progression. Cancer Metastasis Rev 25: 695-705, 2006.

27. Waris G and Ahsan H: Reactive oxygen species: role in the development of cancer and various chronic conditions. J Carcinog 5: $14,2006$.

28. Sotgia F, Martinez-Outschoorn UE and Lisanti MP: Mitochondrial oxidative stress drives tumor progression and metastasis: should we use antioxidants as a key component of cancer treatment and prevention? BMC Med 9: 62, 2011

29. Reuter S, Gupta SC, Chaturvedi MM and Aggarwal BB Oxidative stress, inflammation, and cancer: how are they linked? Free Radic Biol Med 49: 1603-1616, 2010.

30. Elsaleh H, Powell B, McCaul K, et al: P53 alteration and microsatellite instability have predictive value for survival benefit from chemotherapy in stage III colorectal carcinoma. Clin Cancer Res 7: 1343-1349, 2001.

31. Zeng ZS, Sarkis AS, Zhang ZF, et al: p53 nuclear overexpression: an independent predictor of survival in lymph node-positive colorectal cancer patients. J Clin Oncol 12: 2043-2050, 1994.

32. Houbiers JG, van der Burg SH, van de Watering LM, et al: Antibodies against p53 are associated with poor prognosis of colorectal cancer. Br J Cancer 72: 637-641, 1995.

33. Yu SJ, Yu JK, Ge WT, Hu HG, Yuan Y and Zheng S: SPARCL1, Shp2, MSH2, E-cadherin, p53, ADCY-2 and MAPK are prognosis-related in colorectal cancer. World J Gastroenterol 17: 2028-2036, 2011 